Address for Correspondence: Prof. Tian Hui,

Department of Geriatric Endocrinology, Chinese PLA General Hospital,

Beijing 100853, China.

E-mail: tianhui@medmail.com.cn

\begin{tabular}{|l|}
\hline Access this article online \\
\hline $\begin{array}{l}\text { Website: } \\
\text { www.intern-med.com }\end{array}$ \\
\hline DOI: \\
10.1515/jtim-2013-0009 \\
\hline Quick Response Code: \\
\hline \\
\\
\hline
\end{tabular}

\title{
Senile parathyroid dysfunction: Characteristics of its diagnosis and treatment
}

\author{
Shuang-Tong Yan, Hui Tian \\ Department of Geriatric Endocrinology, Chinese PLA General Hospital, Beijing 100853, China
}

\section{ABSTRACT}

Senile parathyroid dysfunction is mainly seen in primary or secondary hyperparathyroidism (pHPT or sHPT). pHPT in the elderly usually occurs in women, with fatigue and osteoporosis as the common symptoms. Screening of blood calcium and further evaluation of parathyroid hormone $(\mathrm{PTH})$ for subjects with serum calcium $>2.6 \mathrm{mmol} / \mathrm{L}$ can be helpful for early identification of patients. For pHPT patients with no contraindications to surgery and whose lesions can be well-localized, surgical treatment can be more beneficial. sHPT is mainly found in patients with end-stage chronic renal dysfunction necessitating hemodialysis or peritoneal dialysis. For these patients, monitoring of PTH level, early supplement of calcium preparations and use of large-dose calcitriol will facilitate maintaining levels of blood calcium and PTH.

Key words: elderly, parathyroid, primary hyperparathyroidism, secondary hyperparathyroidism

\section{INTRODUCTION}

Parathyroid dysfunction, which is relatively rare of the endocrine dysfunction diseases, is, however, common in the elderly, mainly manifested as primary or secondary hyperparathyroidism (pHPT or sHPT). Because pHPT is rare and most are secondary to the total thyroidectomy, the details about it will not be addressed here.

\section{SENILE pHPT}

The pathophysiology of pHPT is mainly osteoporosis and fibrocystic osteitis (manifested as bone pain and multiple fractures) due to an excessive loss of bone calcium induced by high levels of parathyroid hormone (PTH). As a result of the increasing popularity of tests for serum calcium and serum PTH, early identification could be achieved in more patients with asymptomatic hyperparathyroidism.

\section{Epidemiology}

Patients with $\mathrm{pHPT}$ could be seen in all age groups, with a peak incidence in 50-60 years old group according to reports abroad and a female-to-male ratio of $3: 1$ to $4: 1$. The patients were 32-42 years old in previous reports in our country, but the elderly, especially the postmenopausal women have taken a significantly increased proportion in patients with $\mathrm{pHPT}$, possibly related to the degree of aging and enhanced screening efforts for $\mathrm{pHPT}$. According to the report by Jorde et al. ${ }^{[1]}$ in 2000, the prevalence was $13.9 \%$ in women aged $50-75$ years, whereas the prevalence of $\mathrm{pHPT}$ in older men reduced greatly when compared with women. In a screening for hypercalcemia by serum calcium among 2451 middle-aged and old people (with a male proportion of $82.9 \%$ ) receiving health check in our hospital, the author tested PTH in 83 individuals $(3.39 \%)$ with serum calcium $\geq 2.6 \mathrm{mmol} / \mathrm{L}$ and found only 5 with serum PTH $>65 \mathrm{ng} / \mathrm{L}$, indicating that the detection rate of pHPT was $0.204 \%$ in this elderly population, excluding other secondary factors contributing to PTH increase. ${ }^{[2]} \mathrm{A}$ study by Siilin $e t a l l^{[3]}$ tested several indicators including serum calcium, serum PTH and bone density in 3014 older men (those with renal insufficiency and decreased levels of vitamins were excluded) from an area of Sweden. Their results showed that the 
prevalence of parathyroid dysfunction was about $0.73 \%$ in this population and the bone density of patients' collum femoris and buttock significantly decreased in comparison with that of the normal population $(P<0.05)$.

\section{Clinical features}

Osteoporosis and recurrent urinary tract calculi are typical clinical features of $\mathrm{pHPT}$, which is also manifested by other non-specific symptoms including polydipsia, polyuria, weakness, fatigue, abdominal distension, anorexia, constipation, as well as mental status change. More and more asymptomatic patients have been identified since the serum calcium became a routinely tested indicator in 1970s. The elderly take a rather high proportion in patients without typical symptoms, which has caused some difficulties to the diagnosis of $\mathrm{pHPT}$ in the aged people.

Most patients will not visit the hospital for symptoms including fatigue, weakness, anorexia and constipation, which is considered as normal situations of aging. Mental status change is commonly seen in senile patients with pHPT and manifested by significantly impaired memory, depression, irritability and personality changes, which are, however, also clinical symptoms usually found in most aged people and of no specificity. According to an investigation by Politz and Norman ${ }^{[4]}$ among 150 pHPT patients aged over 80 years, fatigue (62\%), hypertension $(62 \%)$ and impaired memory (57\%) were their main symptoms. Even the appearance of osteoporosis, bone pain and height shortening will be naturally regarded as "age-related physiological changes" and lead to delay of diagnosis and treatment. Therefore, the routine test of serum calcium is of key importance to senile patients. When the serum calcium is found increased or close to the upper limit of normal value, serum PTH level should be examined for early identification of $\mathrm{pHPT}$.

The non-specific neuropsychiatric symptoms (e.g., easily fatigue, anxiety, memory decline, inability to concentrate) concomitantly occurred in senile patients with HPT are regarded as the common manifestations of patients with hypercalcemia. Senile HPT is also commonly complicated by hypertension and abnormal glucose and lipid metabolism. The pathogenesis of concomitant hypertension is not yet clear and it is considered attributed to the certain relationships with the vasodilation and impaired renal function due to hypercalcemia. For mechanisms underlying the abnormal glucose and lipid metabolism, it considered to be related with the inherent insulin resistance in patients with HPT. ${ }^{[5]}$

Most patients with primary HPT also suffer hypercalcemia. Wills first introduced the concept of 'normocalcemic pHPT'. In a study done by Silverberg et al. performed a follow-up study among 22 patients with normocalcemic
pHPT for 1 year, and found an increase of serum calcium in 3 of them. A total of 35 patients with normocalcemic pHPT have been reported in our country since 1985. The most effective methods for localization include ${ }^{99 \mathrm{~m}} \mathrm{Tc}$ scanning (methoxy-isobutyl-isonitrile [MIBI]), computed tomography, magnetic resonance imaging and ultrasonic examination. MIBI has be considered the most sensitive and effective method for parathyroid localization, with a positive rate of over $90 \%$ according to reports at home and abroad. As a recently developed detection method, intraoperative B-mode ultrasound has been proved valuable to $\mathrm{PHPT}$ patients with negative MIBI scanning. According to a trial in which the intraoperative ultrasound and MIBI examination were performed among $180 \mathrm{pHPT}$ patients who received limited parathyroidectomy, intraoperative ultrasound examination showed better results in 23 of the 36 patients that could not be accurately positioned by MIBI $(20 \%)$ and raised the localization accuracy from $80 \%$ to $90 \%(P<0.01) .{ }^{[6]}$

\section{Treatment of senile hyperparathyroidism}

\section{Surgical treatment}

Surgery is the most precise and effective treatment of pHPT. Most of studies have proved that surgical treatment is safe for senile patients. "Minimally invasive treatment" is a recently developed the new technique. The specific procedures include preoperative localization of parathyroid lesions by detection methods such as ${ }^{99 \mathrm{~m}} \mathrm{Tc}$ radioisotope scanning, a further intraoperative localization of parathyroid lesions by rapid PTH assay to avoid surgical exploration of normal parathyroid tissues. Uden et al..$^{[7]}$ in their study compared the difference between the safety of surgical treatment in senile patients and young patients. The researchers divided the HPT patients who received surgical treatment into two groups according to their age: Group of 119 patients aged less than 60 years old and the other group of 131 aged over 60 years old. Statistical results found no statistical significance in success rate of surgery between the two groups, $83 \%$ of the young patients and $82 \%$ of the senile patients feeling significantly improved after surgery in comparison with their symptoms before surgery, zero surgery-related mortality in both groups and also an extremely low incidence of postoperative complications were found (temporary vocal cord paralysis occurred to one patient and transient hypocalcemia was developed in 2 of the young patients and 3 of the senile patients).

There have been controversies as to whether the surgical treatment should be applied to patients with asymptomatic hyperparathyroidism, especially the senile patients. It was previously considered that most of the senile HPT patients presented a "benign" progression clinically. Rohl et al. performed a clinical observation among 30 patients with $\mathrm{pHPT}$ who received no surgical treatment 
due to the senility or lacking of symptoms and 15 patients without remission of hypercalcemia after surgery and found no further development of clinical or biochemical manifestations in all patients for 3 years. Thus, the author concluded that long-term conservative treatment instead of surgical treatment could be effective in most patients with asymptomatic hyperparathyroidism. However, increasing evidence in recent years has proved high cardiovascular disease mortality and a high incidence of abnormal metabolism in pHPT patients, which can even influence the overall survival of patients. Therefore, a lot of studies have suggested that surgical treatment should be performed on HPT patients with mild symptoms (mainly the senile patients) if the localization could be achieved and the patient is surgically tolerant. A 6-year follow-up study of 5 pHPT patients identified in our hospital showed that burnout and osteoporosis were the main clinical manifestations, and levels of serum PTH increased 1.5-3 folds continuously, with no spontaneous remission found in any of the cases. All five cases were complicated by cardiac insufficiency, multiple artery calcifications, hypertension and abnormal glucose and lipid metabolism and 2 cases complicated by multisystem disease deceased. One patient hesitated to have surgery at first, and later was considered inoperable due to the senility. The other two patients with atypical symptoms who refused surgical treatment were still followed-up. The 2002 guidelines for asymptomatic pHPT by NIH in the United States loosened indications for surgery in comparison with the 1990 version. ${ }^{[8]}$ The guidelines mentioned: (1) Serum calcium above the normal value of $27.5 \mathrm{mmol} / \mathrm{L}$; (2) 24-h urine calcium $>400 \mathrm{mg}$; (3) decrease of creatinine clearance $>30 \%$; (4) bone density at any site with a reduction of more than 2 standard deviations; (5) aged less than 50 years old; (6) inability to continue follow-up; and (7) patient's requirement for surgery. Indication for surgery will be considered as long as any of the above is met. Meanwhile, once the localization could be achieved in patients with asymptomatic pHPT, timely surgical treatment will be more beneficial even if the requirements for surgery are not met, unless there is a contraindication to surgery. However, based on the summary of multiple-year clinical observation, the 2008 guidelines only recommended surgical treatment of asymptomatic $\mathrm{pHPT}$ for patients with complications of kidney calculi, urine calcium over $10 \mathrm{mmol} / \mathrm{d}$ (or $400 \mathrm{mg} / \mathrm{d}$ ) and/or $T$ value of bone density at the lumbar spine, $1 / 3$ hip and distal of the radius $\leq 2.5 .{ }^{[9]}$ This is because the decrease of bone density in some patients with asymptomatic pHPT could not be observed until 8-10 years later, and some patients had a normal calcium level and a slight increase of PTH. Therefore, it is considered that the data about the incidence and disease progression in such patients are still limited and some patients do not need a surgical treatment.
Drug treatment

For inoperable pHPT patients, drugs can be used for symptomatic treatment. Senile patients with atypical clinical symptoms have better compliance with oral drugs. Drugs including estrogen, raloxifene and "calcium-simulating agent" could be alternatives for treatment of senile primary HPT. Raloxifene, an estrogen receptor modulator, is effective for treatment of osteoporosis in old women and could significantly reduce the levels of bone resorption indices and urine calcium excretion in these patients. "Calcium-simulating agent" achieves good therapeutic outcomes by simulating the effect of calcium on the extracellular calcium receptors, and could significantly reduce the levels of serum calcium and PTH in patients with pHPT. Diphosphate preparations take effect by inhibiting osteoclast activity. Alendronate sodium has been proved potent in increasing bone density by a 12 -month double - blinded, randomly controlled study with a dose of $10 \mathrm{mg} / \mathrm{d}^{[10]}$

\section{SENILE sHPT}

Senile sHPT is mainly found in patients with end-stage chronic renal insufficiency needing hemodialysis or peritoneal dialysis.

\section{Pathogenesis of sHPT}

sHPT is one of the common complications of chronic renal failure (CRF). CRF could cause elevated level of serum phosphorus (decrease of renal clearance) and declined activity of dihydroxyvitamin D3 (hydroxylation defect of $\alpha 1$ ), lead to hypocalcemia, induce sHPT and also contribute to renal osteodystrophy. About $67 \%$ of CRF patients are complicated by sHPT, with a significantly higher incidence and severity in middle-aged patients, especially in those who received a kidney transplant treatment and $6-7 \%$ of the patients developed tertiary HPT after kidney transplantation. In senile patients, however, sHPT is mainly found in CRF patients who need hemodialysis or peritoneal dialysis. Hypertension and diabetic nephropathy cause about half of the CRF in old people, which is higher than the contribution to $\mathrm{CRF}$ in the young and the middle-aged (mainly caused by glomerulonephritis). Senile CRF patients have increased risk for macroangiopathy, and concomitant sHPT could aggravate the progression of atherosclerosis.

\section{Drug treatment of senile sHPT}

For treatment of senile sHPT, appropriate supplement of calcium and calcitriol could maintain an adequate level of calcium during dialysis in most cases $^{[11]}$ and the oral dose usually recommended is $1.2-1.5 \mathrm{~g} / \mathrm{d}$ for calcium carbonate and $0.25-1.5 \mu \mathrm{g} / \mathrm{d}$ for calcitriol. In 2006 , the 'clinical practice guidelines for bone metabolism and disease in chronic 
kidney disease (CKD)' working group suggested that measurement of PTH should be initiated when glomerular filtration rate is found lower than $60 \mathrm{~mL} /\left(\mathrm{min} \bullet 1.73 \mathrm{~m}^{2}\right)$ (CKD stage III) ${ }^{[12]}$ Administration of $0.25 \mu \mathrm{g} / \mathrm{d}$ calcitriol should be started with serum calcium $<2.37 \mathrm{mmol} / \mathrm{L}$ and serum phosphorus $<1.49 \mathrm{mmol} / \mathrm{L}$, followed by regular measurement (every other 3 months) of serum calcium, phosphorus and PTH. Calcium-phosphorus product should be kept below 55. The dose of calcitriol could be adjusted according to the PTH level with the aim of decreasing serum PTH level into the range of 16.5-33.0 $\mathrm{pmol} / \mathrm{L}$. The dose of calcitriol could be increased by $25-$ $50 \%$ based on the original dose for cases with serum PTH $>33.0 \mathrm{pmol} / \mathrm{L}$; the dose is kept unchanged for serum PTH within $22-33.0 \mathrm{pmol} / \mathrm{L}$; it could be reduced by $25-50 \%$ of the original dose for serum PTH within $16.5-22.0 \mathrm{pmol} / \mathrm{L}$; serum PTH $<16.5 \mathrm{pmol} / \mathrm{L}$, serum calcium $>2.54 \mathrm{mmol} / \mathrm{L}$ and serum phosphorus $<1.78 \mathrm{mmol} / \mathrm{L}$ are indicators for calcitriol withdrawal, which usually lasts 3 months first, followed by re-examination of the indicators to decide whether calcitriol should be administered again.

\section{Other treatments of senile sHPT}

Parathyroidectomy surgery will be needed due to the ineffective drug treatment in 5\% of the young and middleaged patients with sHPT, of whom most are those who received kidney transplantation. Kidney transplantation recipients account for a small proportion in senile patients with CRF, and those needing parathyroidectomy due to sHPT are also fewer in senile patients than in the young and middle-aged patients with CRF. Luo et al. ${ }^{[13]}$ conducted B-mode ultrasound-guided percutaneous ethanol injection therapy (PEIT) among 26 hemodialysis patients with sHPT complicated by parathyroid adenoma with a diameter of $>1.0 \mathrm{~cm}$, a certain degree of reduction in levels of serum PTH, serum phosphorus and akaline phosphatase was found in 15 of the patients during a 3 -year observation. PEIT is relatively simple and safe, and it could be considered as an alternative for the treatment of patients with senile sHPT which is difficult to control by drugs, based on the rapid development of interventional B-mode ultrasound.

\section{REFERENCES}

1. Jorde R, Bonaa KH, Sundsfjord J. Primary hyperparathyroidism detected in a health screening. The Tromso study. J Clin Epidemiol 2000;53:1164-9.

2. Yan ST, Tian H, Li CL, Zong WY, Shao YH, Zhong WW, et al. A preliminary survey of primary hyperparathyroidism in middle-aged and elderly Beijing Chinese. Zhonghua Nei Ke Za Zhi 2007;46:651-3.

3. Siilin H, Lundgren E, Mallmin H, Mellstrls D, Ohlsson C, Karlsson $\mathrm{M}$, et al. Prevalence of primary hyperparathyroidism and impact on bone mineral density in elderly men: MrOs Sweden. World J Surg 2011;35:1266-72.

4. Politz D, Norman J. Hyperparathyroidism in patients over 80: Clinical characteristics and their ability to undergo outpatient parathyroidectomy. Thyroid 2007;17:333-9.

5. Yan ST, Tian H. Senile primary hyperparathyroidism. Chin J Osteoporos 2006;12:197-200.

6. Solorzano CC, Lee TM, Ramirez MC, Carneiro DM, Irvin GL. Surgeonperformed ultrasound improves localization of abnormal parathyroid glands. Am Surg 2005;71:557-62.

7. Udén P, Chan A, Duh QY, Siperstein A, Clark OH. Primary hyperparathyroidism in younger and older patients: Symptoms and outcome of surgery. World J Surg 1992;16:791-7.

8. Bilezikian JP, Potts JT Jr, Fuleihan Gel-H, Kleerekoper M, Neer $\mathrm{R}$, Peacock $\mathrm{M}$, et al. Summary statement from a workshop on asymptomatic primary hyperparathyroidism: A perspective for the $21^{\text {st }}$ century. J Bone Miner Res 2002;17:N2-11.

9. Silverberg SJ, Lewiecki EM, Mosekilde L, Peacock M, Rubin MR. Presentation of asymptomatic primary hyperparathyroidism: Proceedings of the third international workshop. J Clin Endocrinol Metab 2009;94:351-65.

10. Khan AA, Bilezikian JP, Kung AW, Ahmed MM, Dubois SJ, Ho AY, et al. Alendronate in primary hyperparathyroidism: A double-blind, randomized, placebo-controlled trial. J Clin Endocrinol Metab 2004;89:3319-25.

11. Sprague SM, Coyne D. Control of secondary hyperparathyroidism by vitamin $\mathrm{D}$ receptor agonists in chronic kidney disease. Clin J Am Soc Nephrol 2010;5:512-8.

12. Clinical practice guidelines for bone metabolism and disease in chronic kidney disease. Chin J Blood Purif 2006;5:275-80.

13. Luo Y, Zhang L, Bian WJ, Liu YM, Li C, Li WG, et al. Long-term prognosis of secondary hyperparathyroidism in hemodialysis patients after percutaneous ethanol injection therapy. J Chin Japan Friend Hosp 2007;21:333-6.

How to cite this article: Yan ST, Tian H. Senile parathyroid dysfunction: Characteristics of its diagnosis and treatment. J Transl Intern Med 2013; 1(1): 32-5.

Source of Support: Nil, Conflict of Interest: None declared 